\title{
Development of precision hard x-ray multilayer optics with sub-arcminute performance
}

Jason E. Koglin, Hubert C.M. Chen, Finn Erland Christensen, Jim Chonko, William W. Craig, et al.

Jason E. Koglin, Hubert C.M. Chen, Finn Erland Christensen, Jim Chonko, William W. Craig, Todd R. Decker, Mario A. Jimenez-Garate, Charles J. Hailey, Fiona A. Harrison, Carsten P. Jensen, Mike Sileo, David L. Windt, Haitao $\mathrm{Yu}$, "Development of precision hard X-ray multilayer optics with subarcminute performance," Proc. SPIE 4851, X-Ray and Gamma-Ray Telescopes and Instruments for Astronomy, (11 March 2003); doi: $10.1117 / 12.461488$

SPIE Event: Astronomical Telescopes and Instrumentation, 2002, Waikoloa, Hawai'i, United States 


\title{
Development of precision hard X-ray multilayer optics with sub-arcminute performance
}

\author{
Jason E. Koglin ${ }^{a}$, Hubert C. Chen ${ }^{b}$, Finn E. Christensen ${ }^{c}$, \\ Jim Chonko ${ }^{a}$, William W. Craig ${ }^{d}$, Todd R. Decker ${ }^{d}$, \\ Mario A. Jimenez-Garate ${ }^{e}$, Charles J. Hailey ${ }^{a}$, Fiona A. Harrison ${ }^{b}$, \\ Carsten P. Jensen ${ }^{c}$, Mike Sileo $^{a}$, David L. Windt ${ }^{a}$, Haitao $\mathrm{Yu}^{a}$ \\ ${ }^{a}$ Columbia Astrophysics Laboratory, New York, NY \\ ${ }^{b}$ California Institute of Technology, Pasadena, CA \\ ${ }^{c}$ Danish Space Research Institute, Copenhagen, Denmark \\ ${ }^{d}$ Lawrence Livermore National Laboratory, Livermore, CA \\ ${ }^{e}$ Massachusetts Institute of Technology, Cambridge, MA
}

\begin{abstract}
A new generation of hard X-ray telescopes using focusing optics are poised to dramatically improve the sensitivity and angular resolution at energies above $10 \mathrm{keV}$ to levels that were previously unachievable by the past generation of background-limited collimated and coded-aperture instruments. Active balloon programs $(\mathrm{HEFT}, \mathrm{InFoc} \mu \mathrm{s})$, possible Explorer-class satellites, and major X-ray observatories (Constellation-X, XEUS) using focusing optics will play a major role in future observations of a wide range of objects including young supernova remnants, active galactic nuclei, and galaxy clusters. These instruments call for grazing incidence optics coated with depth-graded multilayer films to achieve large collecting areas. To accomplish the ultimate goals of the more advanced satellite missions such as Constellation-X, lightweight and low-cost substrates with angular resolution well below an arcminute must be developed. Recent experimental results will be presented on the development of improved substrates and precision mounting techniques that yield sub-arcminute performance.
\end{abstract}

Keywords: X-ray optics, Hard X-ray telescope

\section{SCIENCE GOALS AND OPTICS REQUIREMENTS}

A new generation of experiments is necessary to perform sensitive observations on a wide range of astrophysical sources at photon energies above $10 \mathrm{keV}$. These missions require focusing optics to achieve the sensitivity required for the science goals. A variety of approaches have been taken to build soft X-ray mirrors in the past. Aluminum foils (ASCA, ${ }^{1}$ ASTRO-E, ${ }^{2}$ SODART ${ }^{3,4}$ ), electroformed nickel from replication (XMM-Newton ${ }^{5}$ ) and monolithic Zerodur (Chandra ${ }^{6}$ ) have been successfully used as optic substrates. We have choosen to develop a different approach - thermally-formeded, segmented glass optics. We are currently using these optics and our unique mounting approach to build optics modules for the large area High Energy Focusing Telescope (HEFT) balloon born mission.

Relatively large collecting areas are achieved using nested, conic approximation, Wolter-I mirror assemblies that focus X-rays through total external reflection. Since the critical angle (the maximum incidence angle at which total external reflection occurs) is inversely proportional to the photon energy, very small incidence angles are required to reflect high-energy X-rays. Multilayer coatings consisting of alternating layers of high and low density materials can be used to achieve high reflectivities at angles greater than the critical graze angle through constructive interference at multiple surface interfaces. Depth-graded multilayer coatings vary the thickness of these alternating layers to achieve high reflectivity over a broad range of energies, even extenting above 100 $\mathrm{keV} .{ }^{7}$ Some of the primary scientific objectives for HEFT include imaging and spectroscopy of ${ }^{44} \mathrm{Ti}$ emission in

Correspondence: koglin@astro.columbia.edu, http://astro.columbia.edu/ koglin 
young supernova remnants, sensitive observations of obscured Active Galactic Nuclei, spectroscopic observations of accreting pulsars with high-magnetic fields, and mapping the Galactic Center.

Our highest priority for new research on focusing optics is to provide a viable approach for the hard X-ray telescope (HXT) on the Constellation-X (Con-X) satellite mission, a high-resolution spectroscopy mission with large effective area that is under study by NASA as a follow-on to Chandra. ${ }^{*}$ The HXT will provide information on source spectra at higher energies than the Con-X spectroscopy X-ray telescope (SXT). It will independently probe the emission from astrophysically important objects like shrouded AGN, which are now believed to be very significant contributors to the diffuse hard X-ray background. The HXT will also permit high resolution mapping of continuum and line emission in SNR and deep imaging of the galactic center.

Thermally-slumped glass optics, which we have developed, ${ }^{8}$ have become one of the leading candidates for the Con-X hard X-ray telescope (HXT), which requires 1' angular resolution and has a goal of 30". We expect to eventually be able to produce 20" substrates with our forming technique as we will discuss in Section 3 . We also have new ideas for achieving even higher performance that we present in Section 4. Our approach has been modified by others to include an extra epoxy replication step. ${ }^{9}$ These epoxy-replicated glass substrates have the potential to achieve performance down to $5 " 10,11$ and are the current baseline technology for the Con-X spectroscopy X-ray telescope (SXT). Our current mounting approach is far superior to the requirements of HEFT. ${ }^{12}$ In fact, our current mounting approach satisfies the angular resolution goal for the HXT optics. It is flexible enough to accommodate almost any size of segmented shell and has the potential to meet the design criteria for future Explorer class satellites and major space-based observatories. We can likely meet the SXT performance goal by incorporating straightforward modifications into our mounting approach (described in Section 2), many of which we are currently implementing. Thermally-slumped glass is also being considered for XEUS, an ESA mission similar to Con-X but with 5-20 times larger effective area at $1 \mathrm{keV}$. The scale of this mission is staggering and, it is clear that XEUS will require extreme low cost per unit area, rapidly buildable and high performance optics.

\section{MOUNTING TECHNIQUE}

The mounting process we have developed for segmented glass substrates is unique. ${ }^{13,14}$ The glass segments are constrained to precisely machined graphite spacers that run along the optical axis. In this way, the nominally cylindrical glass segments are forced to a conical form, and in the process, small twists and radial mismatches in the glass are removed. At the same time, the good properties of the glass such as small scale figure and microroughness are retained. We have collaborated with Colorado Precision Products, Inc. (CPPI) in Boulder, Colorado to design and build a precision assembly machine for hard X-ray telescopes. We are currently using this machine to build flight optics modules for HEFT, which requires $20 \mathrm{~cm}$ long upper and lower shells with radii between $4 \mathrm{~cm}$ and $12 \mathrm{~cm}$. However, the mounting approach itself is flexible enough to handle almost any length and radius segments. For HEFT, the $20 \mathrm{~cm}$ long upper and lower shells are actually composed of two $10 \mathrm{~cm}$ long segments each. As will be described later in Section 3, we have also used $5 \mathrm{~cm}$ long segments.

The assembly process begins by aligning a mandrel on the assembly machine. Graphite spacers are epoxied onto the mandrel and machined to the correct radius and angle. Segmented mirror segments are then epoxied to the spacers to form the complete upper and lower layers. In this process, very strong, thin, and uniform epoxy bondlines are achieved. After epoxy cure, the next layer of spacers is epoxied to the previous layer of glass. These spacers are machined and another layer of glass is epoxied to the spacers. In a similar way, subsequent layers are added to the optic assembly as shown in Figure 1.

In this assembly process, each layer of graphite spacers is precisely machined with respect to the optical axis, and thus, assembly errors do not stack up. An air-bearing Linear Variable Differential Transformer (LVDT) ${ }^{\dagger}$ build by CPPI was used to perform measurements of an entire set of 25 spacers, the results of which are shown in Figure 2a. The machined spacers have a high frequency peak to valley roughness of about $1.5 \mu \mathrm{m}$. The figure of the glass will not be compromised by the roughness of the machined spacers since the roughness is typically

\footnotetext{
${ }^{*}$ http://constellation.gsfc.nasa.gov

${ }^{\dagger}$ http: //www . coloradoprecision. com/LVDT2.htm
} 

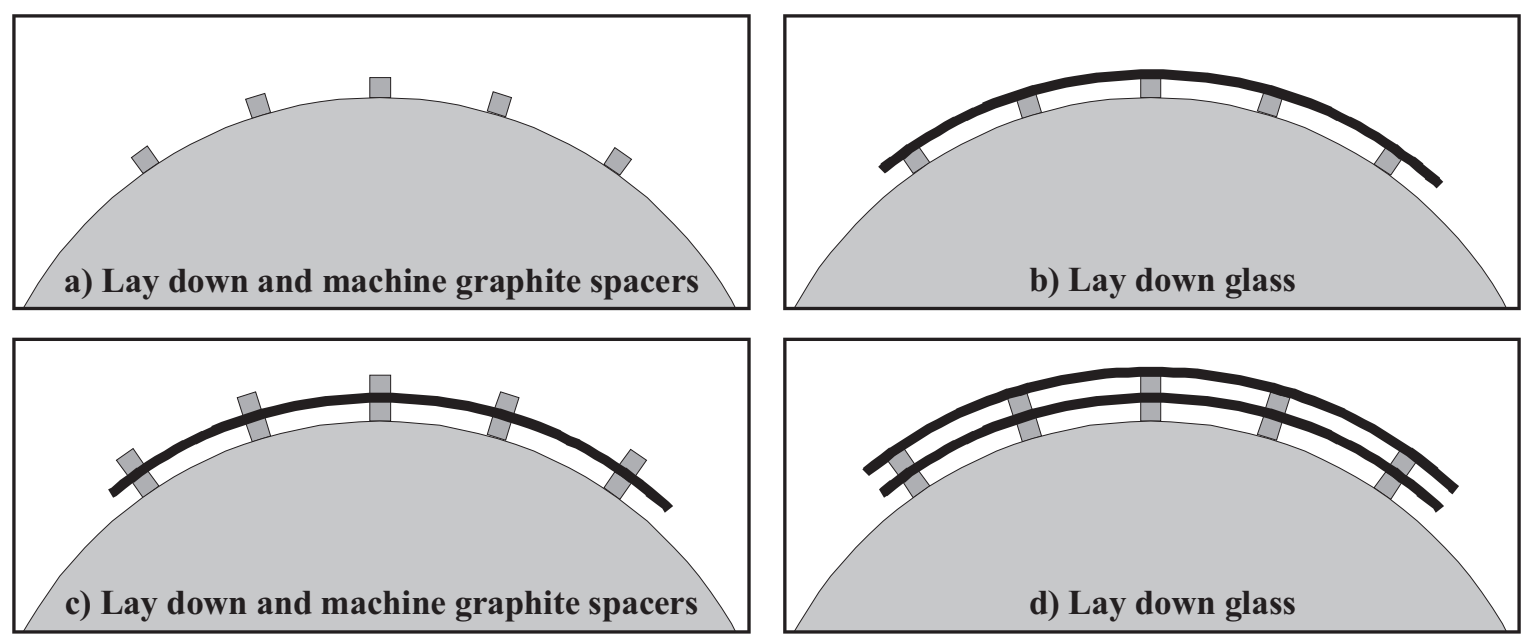

Figure 1. a) The assembly process begins by laying graphite spacers on a precision mandrel and machining them to the correct radius. b) Segmented mirror segments are epoxied to the spacers. c) A new layer of spacers is machined to the first layer of glass and machined to the correct radius. d) The next layer of segmented mirror segments are epoxied to the spacers.
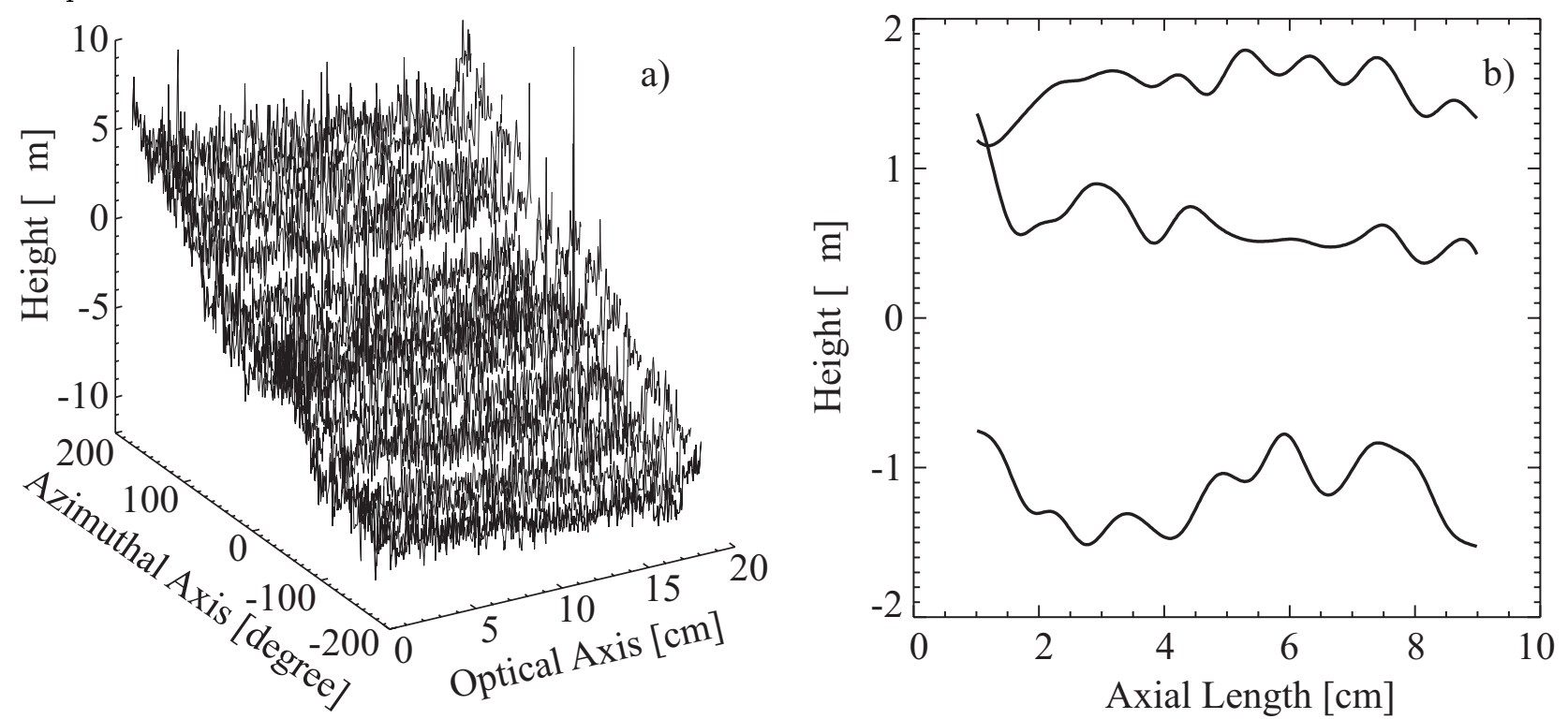

Figure 2. a) LVDT metrology results for a complete set of $20 \mathrm{~cm}$ long machined spacers. b) LVDT metrology of glass on top of three consecutive graphite spacers. A fifth-order Butterworth filter with a frequency cutoff of $1 \mathrm{cycle} \mathrm{per} \mathrm{cm}$ has been applied to the raw data to remove noise introduced during data acquisition.

on a millimeter length scale, much shorter than the scale at which the glass can be deformed. In fact, since this roughness increases the effective surface area of the spacer, it will help increase the strength of the epoxy bond-line. The spacers force the glass to conform to the optical geometry and also remove some of the residual twists present in the slumped glass. The conformance of the glass directly on top of these spacers is superb as is shown in Figure $2 \mathrm{~b}$ for the glass on top of three spacers. Here, the surface height is plotted as a function of the axial length along the spacers. The figure of glass on top of these spacers is 24 ". Much of this can be attributed to the intrinsic figure of the substrate $(\sim 60 ")$ and not to errors in the assembly process. The assembly machine itself is estimated to contribute only about 8 " to the overall error budget. The monolithic structures produced 

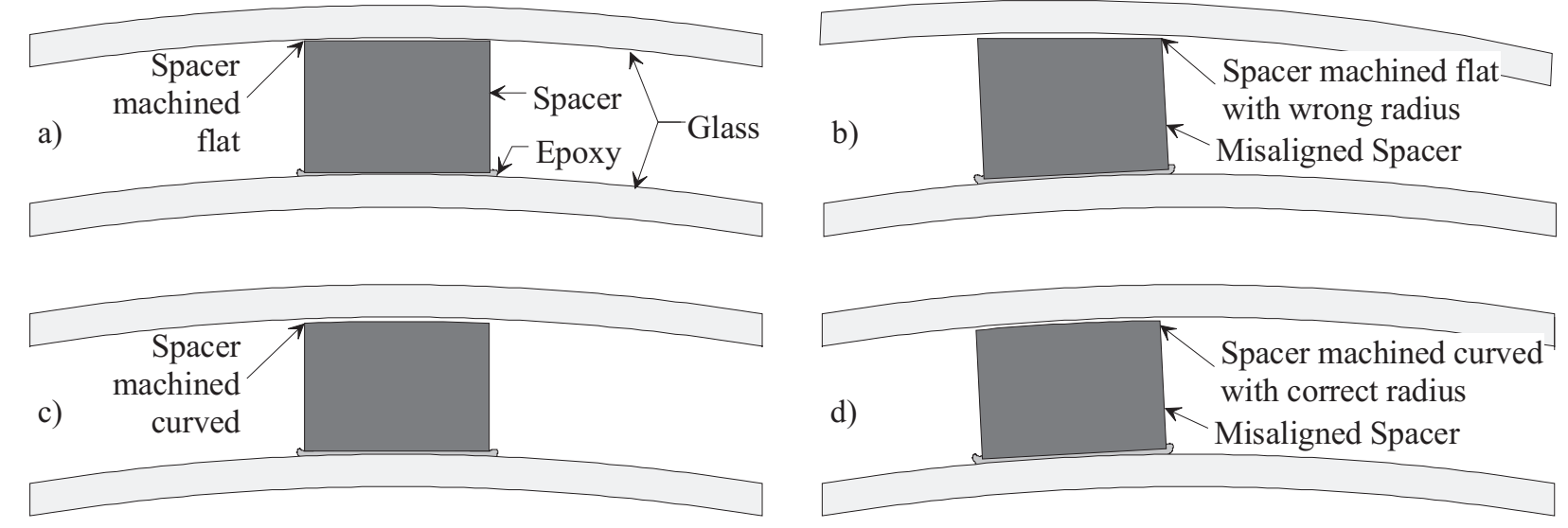

Figure 3. a) The spacer is machined flat and tangential to the optic radius when properly aligned. b) A misalignment in the spacer will cause the spacer to be machined with the wrong radius which may lead to an in-phase roundness error in the mounted shell. c) A spacer is machined uniformly to the correct radius. d) A misalignment in the spacer will have no effect on the spacer performance if the spacer is machined uniformly to the correct radius.

by this method provide the extreme mechanical robustness that is necessary for the instrument to survive both multiple balloon campaigns and satellite deployment.

We have used a deterministic approach to designing and building our telescope assembly machine. In this way, we have a developed a detailed error budget for the assembly process itself and we fully understand the dominant error contributions. The present assembly error contribution is very small compared to that of the HEFT substrates, which are limited by the initial figure of the free-standing mirror segments. However, in anticipation that substrates with figure $\sim 5$ " will be developed in the near future, we will continue to improve the assembly machine to a comparable contribution to overall error budget.

To simplify the assembly process, we approximate the parabolic and hyperbolic shells of a true Wolter-I telescope to be conic shells. In the case of the HEFT design, this leads to an average conical-approximation error of 12". However, with only a moderate modification to the fixturing, the segmented, conic shells could be machined at independent angles. For example, in the current design the conic approximation error could be decreased by a factor of two by machining the spacers at slightly different angles for each of the two $10 \mathrm{~cm}$ long optic pieces that form the upper and lower $20 \mathrm{~cm}$ long segments. Similarly, if four $5 \mathrm{~cm}$ long optic pieces were used to form the $20 \mathrm{~cm}$ long shell, then the conic approximation could error be decreased by a factor of four to just 3". Further, by completely automating the mechanical setup with full computer control, the spacers could be machined to arbitrary shapes including the hyperbolic and parabolic curves necessary for a true Wolter-I design. In principle, the conic approximation error can be eliminated altogether.

A leading assembly error term is the physical error in the azimuthal placement of the spacers. The original machine design calls for the spacers to be machined flat and tangential to the optic radius when properly aligned as illustrated in the cartoon in Figure 3a. However, azimuthal misalignments of the raw spacers (1.6 $\mathrm{mm}$ thick) result in a change in the radius that may cause an in-phase roundness error in the subsequent shell (see Fig. 3b; note that the effect is exaggerated in this cartoon). If the spacer is twisted so that it is not aligned with the optical axis, then the machined radius will effectively vary along the optical axis and lead to a slope error in the shell. We are currently upgrading our assembly fixturing to assure that the spacers are more accurately aligned. Further, we have demonstrated the capability of machining the spacers uniformly to the correct radius as illustrated in Fig. 3c. With this machining method, the spacer is uniformly machined to the correct radius even if the spacer is misaligned (see Fig. 3d). With these modifications, we can reduce this sort of error contribution to less than 1 ".

Another group of systematic errors involves the overall calibration of the machine including verification of the angle and radius of the spacer cut. These errors are the result of performing the verification metrology 
with the same instruments that are used to set the angles and positions of the machine. We plan to implement a fully-independent metrology station in order to reduce systematic errors that now dominate measurements of the spacer positions. This will allow absolute verification of spacer radial and axial profile to the $0.25 \mu \mathrm{m}$ (sub-arcsecond) level. Thermal stability at the level of one degree for the entire setup is necessary in order to improve the figure of the machined spacers as well as minimize measurement and calibration errors. To achieve this, the entire assembly apparatus will be enclosed in a thermally stabilized room. We already have a dust collection system that helps eliminate particulate contamination of the optics that can result in loss of effective area, compromise the performance and integrity of the epoxy bond joints, and limit the reliability of the mechanical metrology. The thermally stabilized room will be designed to provide an even cleaner environment for the assembly production.

With these improvements and modifications to the assembly machine, we expect to reduce the total assembly error budget to less than 3". With improved substrates, this will allow telescopes to be built with < 5" resolution. While this high performance is necessary for future high resolution missions, these missions will also require high throughput, and thus, high performance in itself it is not sufficient. Speed of assembly is crucial. Because of the repetitive nature of the production procedure, small changes in the efficiency in steps in the mounting process can have a huge impact on the overall production rate. In particular, we will implement an automated epoxy applicator in order to achieve a faster as well as more uniform and controlled epoxy application. We will also upgrade the computer and data acquisition system to fully automate metrology and machining of spacers.

Further improvements in efficiency can be made by building telescope modules in parallel. The optic mandrel can be quickly and accurately aligned on the assembly machine, and it is also quick and easy to unmount and accurately remount an optic module. We have performed tests and verified that the accuracy in unmounting and remounting an optic is currently limited by the accuracy of the alignment pin that is required to maintain the alignment of the optic, even when no mounting/unmounting is required. Since the assembly machine is only required to machine the spacers, the optic mandrel can be removed from the machine after the spacers are machined, and the glass segments can be assembled on a separate and much simpler apparatus. During the time necessary to assemble the glass segments on the first mandrel and allow the epoxy to cure, a second optic mandrel can be mounted on the assembly machine and its spacers can be machined. In this way, multiple optic units can be assembled in parallel using the same machine. A great amount of flexibility in accommodating different sizes and types of substrate is also inherent in this method. The assembly apparatus design can be modified to accommodate an optic module with an arbitrarily large radius. Concentric multi-mandrel designs can be used to reduce risk during the production process and improve production efficiency by dedicating separate machines to building different radial shells of the telescope.

\section{SUBSTRATE DEVELOPMENT}

We have also been involved in a vigorous effort to develop thermally-formed glass substrates to be mounted using our assembly approach. We begin with microsheet glass that has good initial properties such as microroughness, figure, and thickness uniformity. These substrates, which were developed for the flat panel display industry, can be made very thin and thus lightweight. The glass has a smooth surface that is ideally suited for multilayer deposition and the low surface roughness of $\sim 3.5 \AA^{15,16}$ provides good x-ray reflectivity even for energies over $100 \mathrm{keV}$. The small scale figure errors intrinsic to this glass will ultimately limit the angular performance to about 8-10 arcseconds.

Our approach is to thermally form these micro-sheets into cylindrical segments using standard quartz mandrels and commercially available ovens. We begin by placing a glass micro-sheet on the mandrel inside of the oven. As the oven is heated to the appropriate forming temperature, the glass begins to form under the influence of gravity. The glass slowly assumes the large-scale figure of the mandrel. Just before the glass touches the mandrel surface, the forming process is terminated by lowering the oven temperature. In this way, near net shaped optic substrates are produced without perturbing the excellent initial X-ray properties of the glass micro-sheet. This allows us to avoid using highly polished and very expensive mandrels.

Fast and accurate metrology evaluation is integral part of our optics development program. A laser scanning metrology apparatus was designed and built at Columbia's Nevis Laboratory and is described more completely 
elsewhere. ${ }^{17}$ From axial scan measurements at multiple azimuthal positions, the cylindrical surface can be fully reconstructed using software that removes any error in the shell alignment. We have also designed and built mounting fixtures for both flat and cylindrical glass substrates. These fixtures serve as surrogates for the mounting process at CPPI and provide a more cost effective approach to optics development research that can be performed in our university laboratory. The surrogate mounts have precision machined spacers against which test substrates can be mounted to simulate the full fabrication process either with or without epoxy (i.e., non-destructive), and the laser scanning apparatus is used to obtain immediate evaluation of the substrate performance. With this approach, we have studied the properties of mounted substrates as a function of compression load and size of substrate. We have further developed and improved our glass mounting procedures and have gained valuable insight into fundamental charateristics of the glass and the mounting process using these surrogate mounting fixtures. An important investigation involved using the laser scanner and the flat mounting fixture to measure and compare the front and back surfaces of flat glass samples. From these measurements, the glass is found to be uniform in thickness such that the front and backsides of the glass have nearly identical large and small scale features (within $\sim 5 "$ ). Additional data have been recorded using both the flat and cylindrical surrogate mounting fixtures. Using these results, we have adapted and modified our techniques and procedures to maximize our potential for sucess in the prototype development work conducted at CPPI. This prototype developtment work will be the focus of the rest of this section.

A full two-bounce prototype optic was initially built to evaluate the assembly process and compare metrology techniques. For this prototype, five $20 \mathrm{~cm}$ long quintant segments where used to make up each upper and lower layer. These conic segments had a nominal diameter of about $16 \mathrm{~cm}$ with each $300 \mu \mathrm{m}$ thick shell subtending about 70 degrees (i.e., an arclength of about $10 \mathrm{~cm}$ ). Five $16 \mathrm{~cm}$ graphite spacers were used to overconstrain each shell. The performance of this optic was characterized using three independent metrology methods: direct metrology performed on the back surface of the segments, full illumination of the entire optic with UV radiation, and multiple $8 \mathrm{keV}$ X-ray pencil beam scans performed over the entire optic. Each of these methods yielded consistent results. The general mounting approach was found to be very effective at constraining the glass substrates along the graphite spacers. The glass tends to return to its original freestanding state away from the spacers. While the central section of these substrates in their unmounted state have relatively good performance, the $\sim 2 \mathrm{~cm}$ at both top and bottom of the glass yield much poorer performance because they naturally tend to curl during the thermal forming process. In this way, the image formed by these mounted shells has a sharp peak that is necessary for resolving close objects. However, the edges of the glass cause a large number of photons to be scattered to the wings of the image, thus degrading the overall HPD performance. A more detailed description of the tests performed on this optic is given elsewhere. ${ }^{12}$

Our scribe and break cutting technique was refined to allow us to cut the formed piece along the curved azimuth direction in order to remove the poor performing edges of the glass. A prototype optic was built using $300 \mu \mathrm{m}$ thick glass substrates of both AF45 and D263 glass. The original 15 or $20 \mathrm{~cm}$ long slumped segments were cut to 35 degree segments, $10 \mathrm{~cm}$ in length. A total of 34 individual segments were mounted in a two-bounce configuration. Axial LVDT scans were performed on each segment every $2.5^{\circ}$, and a fifth-order Butterworth filter with a frequency cutoff of 1 cycle per $\mathrm{cm}$ was been applied to the raw data to remove noise introduced during data acquisition. As an example, the LVDT data for a set of four segments forming a two-bounce section are plotted in Figure 4a. The two rightmost $10 \mathrm{~cm}$ segments form the upper layer of the telescope, the two leftmost segments form the lower layer as indicated in the plot. The focal length of this and the other prototypes that will be discussed later are all $6 \mathrm{~m}$. The first and last segments are shown in the three-dimensional plots in Figures $4 \mathrm{~b}$ and $4 \mathrm{c}$, respectively. The graphite spacers run axially at the azimuthal angles $0^{\circ}, 15^{\circ}$ and $30^{\circ}$. An assembly error is evident for the segment in Figure $4 \mathrm{~b}$ along the spacer located at $0^{\circ}$. It is likely that a particulte contaminated the epoxy bondline causing the glass to be deformed over $\sim 2 \mathrm{~cm}$ on top of the spacer. This mounting error, however, is isolated to a small region of the glass surface and is quickly attenuated as the glass assumes its intrinsic shape away from the spacer. In this way, mounting errors only slightly degrade the performance of a segment. Further, this prototype was assembled before our dust collection system was fully implemented and we now have improved process control such that this type of error has been virtually eliminated.

A histogram of the performance results extracted from LVDT measurements of the 34 individual segments 

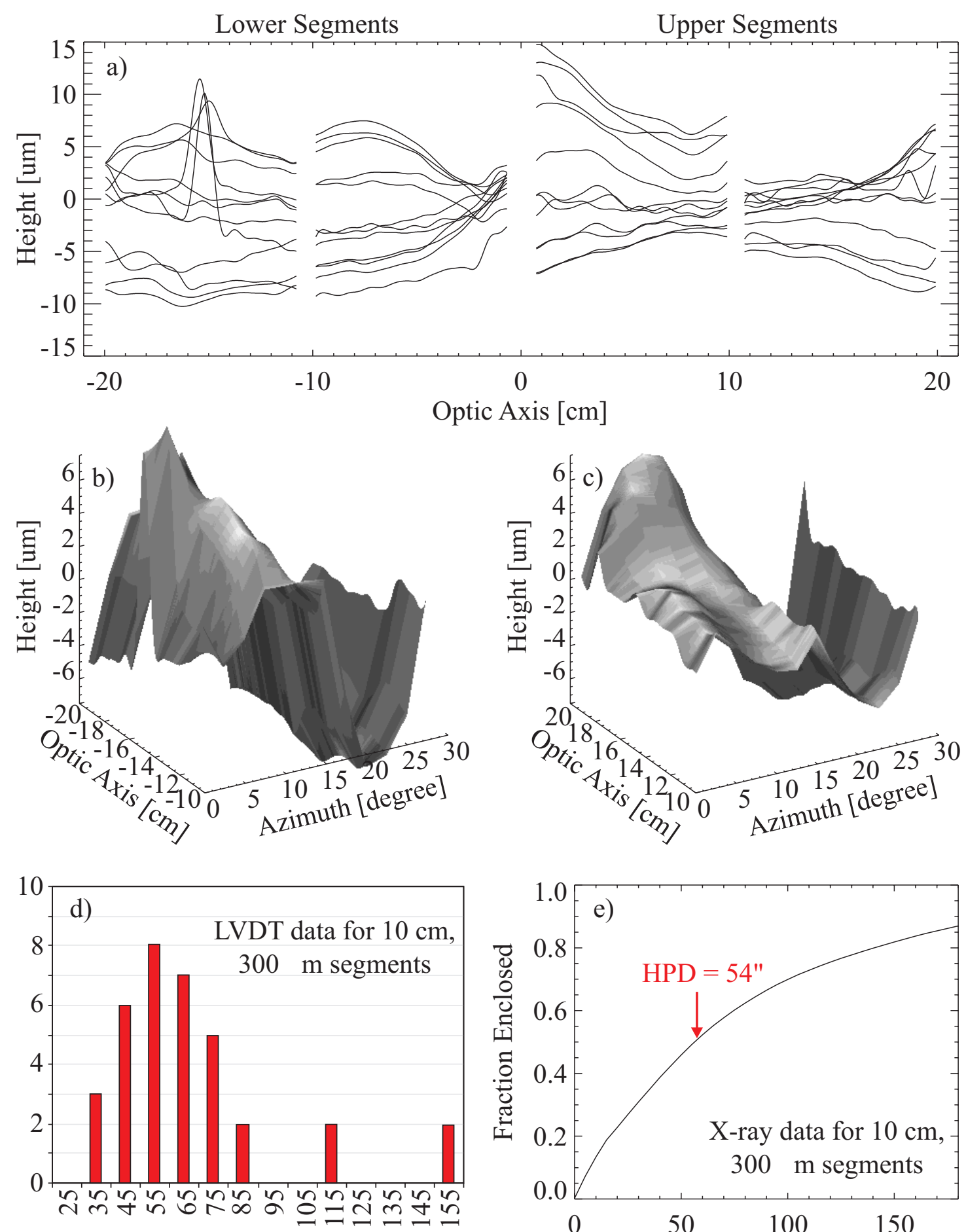

Performance [arcsec]

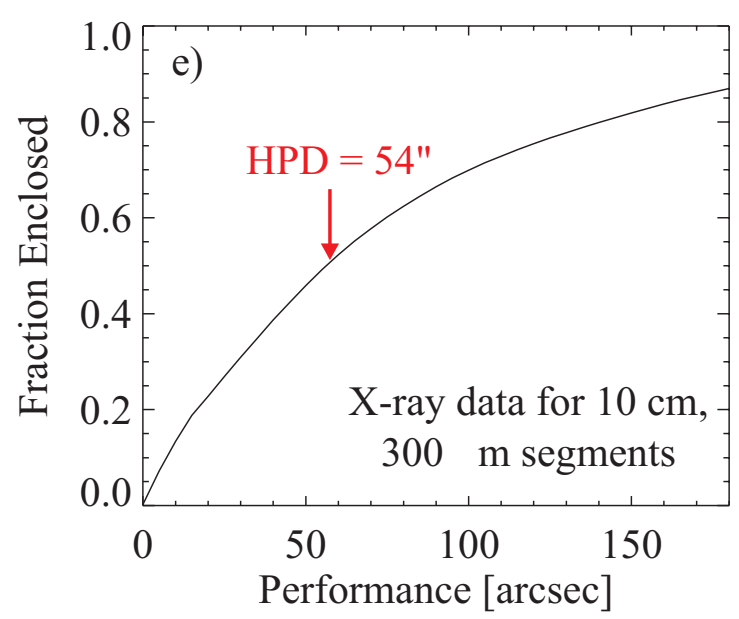

Figure 4. The LVDT axial scans, performed in $2.5^{\circ}$ steps, are plotted in a) for a set of four segments, $300 \mu \mathrm{m}$ thick and $10 \mathrm{~cm}$ long, which form a two-bounce section. Three-dimensional surface plots of the first and third segments are shown in b) and c), respectively. A histogram of the HPD performance results (for 2-bounce optics) determined from LVDT surface metrology data is plotted in d) for the 34 segments of AF45 and D263 glass cut to $10 \mathrm{~cm}$ in length. From the image obtained from the X-ray data, the fraction of enclosed energy is plotted in e) versus performance for the entire optic composed of 34 segments - the HPD of $54 \pm 5 "$ is indicated. 
is shown in Figure 4d. The LVDT data provide an immediate and reliable characterization of the mounted segments that is extremely valuable for quality assurance. It further provides the most detailed information on the individual glass segments and is very useful in understanding the over-constraint mounting process. However, X-ray metrology gives a more accurate determination of the two-bounce performance of an ensamble of shells since the X-ray measurements have very low background and only minimal data analysis is required (no filtering or raytrace simulations are required as with the LVDT data). The fraction of enclosed energy from the X-ray measurements is plotted versus performance for this entire prototype optic in Figure 4c. The HPD performance for this optic is $54 \pm 5$ ", where the uncertainty indicates the systematic aligment error in the X-ray measurement. This length and type of substrate has been adopted for the first HEFT optics modules.

A smaller prototype was also built using $200 \mu \mathrm{m}$ thick glass substrates. LVDT metrology data for an upper and lower set of such segments are shown as an example in Figure 5a. The three-dimensional surface of the lower segment is shown in Figure 5b. The HPD performance of this optic determined from X-ray measurements is $58 \pm 5$ ", as indicated in the enclosed energy versus performance plot in Figure $5 \mathrm{c}$. The performance of these thinner substrates meets the Con-X HXT angular resolution requirement. In addition, these substrates also meet the severe weight restrictions of this mission. An additional small prototype was built using even thinner $100 \mu \mathrm{m}$ and $145 \mu \mathrm{m}$ glass substrates. These thin substrates along with some $300 \mu \mathrm{m}$ thick substrates were the first substrates formed using an alternative thermal forming technique as part of our developmental research program. This technique currently produces substrates with performances not better than 1' and are nearly independent of the substrate thickness. This prototype primarily demonstrates that it is possible to handle and mount such thin pieces of glass. Since the surface figure and roughness specifications for this very thin glass are similar to the thicker $200 \mu \mathrm{m}$ and $300 \mu \mathrm{m}$ glass formed using more developed forming techniques, it is reasonable to believe that with further development, sub-arcminute performance can also be achieved with these substrates.

The performance achieved by our method of thermally forming glass is almost completely limited by the axial bows that occur during the thermal forming process. In this way, substrates cut to shorter lengths are expected to yield correspondingly better performance. To demonstrate this, a small prototype optic was build using $300 \mu \mathrm{m}$ thick glass segments cut to $5 \mathrm{~cm}$ in length. The LVDT data for these four segments, which were mounted in one two-bounce segment, are shown in Figure 6a. The three-dimensional surface of the uppermost shell is shown in Figure 6b. Note that there is a relatively large in-phase roundness error (how the mean height of each axial scan varies with azimuthal angle) in these shells. While the in-phase roundness error is suppressed by the ratio of the radius to the focal length (compared to the axial figure), it begins to contribute to the HPD for these shells. This sort of error can be reduced to achieve even better angular resolution by better matching the radius of the slumped segments to the mounted radius. X-ray metrology was also performed on this prototype. The fraction of enclosed energy is plotted versus performance in Figure $6 \mathrm{c}$ for this prototype the HPD of $45 \pm 5 "$ is indicated.

\section{FUTURE SUBSTRATE DEVELOPMENT}

Based on our optics development program, we have a much greater understanding of how to evaluate substrates used with our overconstraint mounting technique. We have found that surface metrology of a sample of freestanding segments can be used to accurately predict the average performance of an ensemble of mounted segments. With further refinements in the evaluation of the this data, we will be able to more accurately predict the mounted performance on a shell by shell basis. This will allow us to place much more stringent acceptance criteria on the slumped glass. Since we can produce large quantities of relatively inexpensive substrates, the opportunity to rationally 'cherry-pick' becomes a viable means to obtain superior performance. In this way, we expect to be able to achieve the 30" goal of the Con-X HXT using the 'lightweight' glass $(200 \mu \mathrm{m})$ required to meet the HXT weight requirement.

Our future work is as follows. Firstly, we will continue our work on the current approach to producing optics. Secondly, we would like to build on the successes of our current assembly machine to a performance level commensurate with the requirements of future high throughput missions. We are also pursuing new approaches to forming substrates. For example, our team members at LLNL have an ongoing program to 

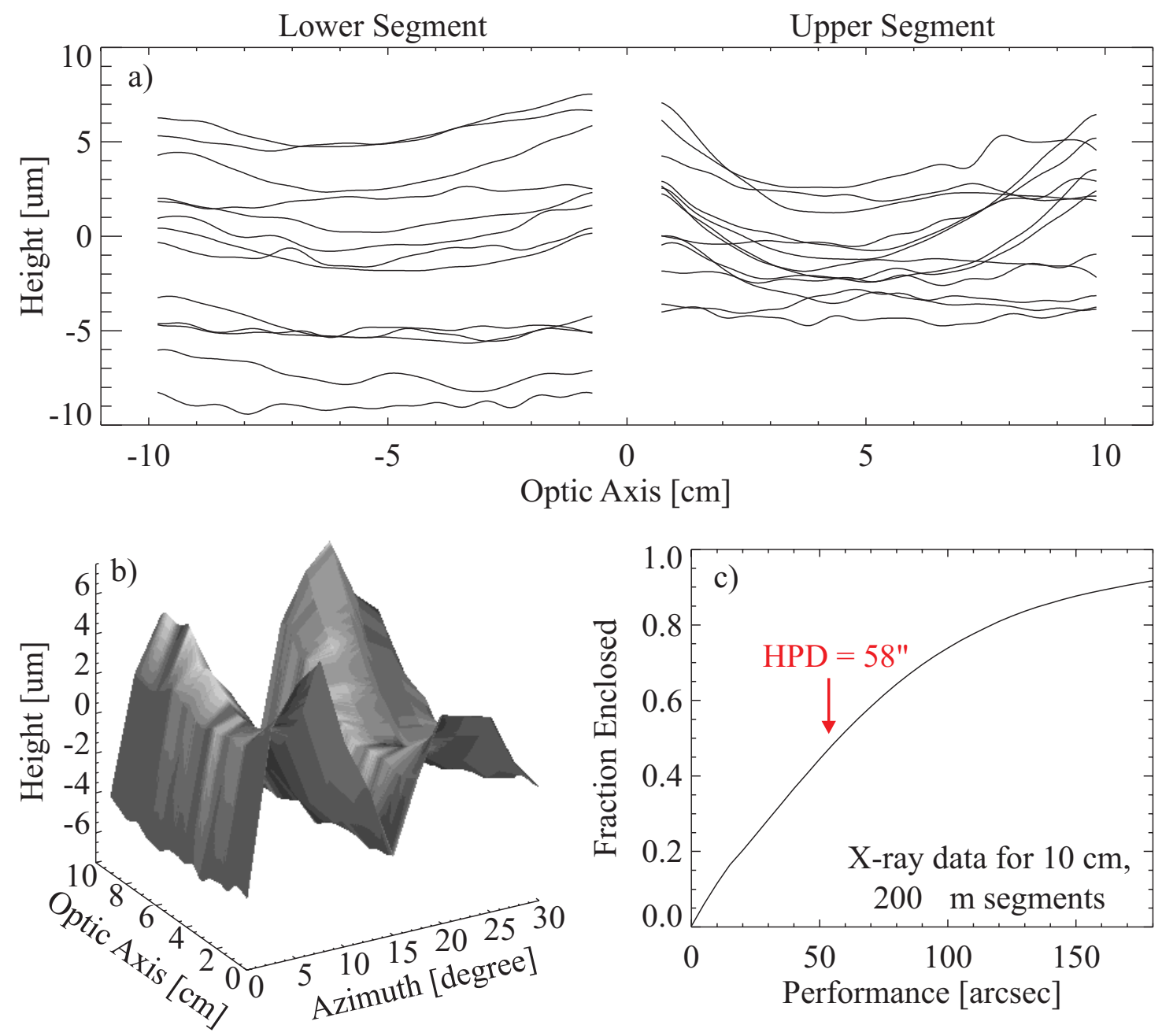

Figure 5. a) LVDT axial scans performed every $2.5^{\circ}$ for a set of two segments, $200 \mu \mathrm{m}$ thick and $10 \mathrm{~cm}$ long, which form a two-bounce section. b) The surface of the lower segment is plotted in three-dimensions. c) From the image obtained from the X-ray data, the fraction of enclosed energy is plotted versus performance for the entire optic composed of 6 segments, and the HPD of $58 \pm 5 "$ is indicated.

develop X-ray optics for medical imaging applications. That program has an eventual goal of 1" with a near term requirement of 20 ". They are pursuing new techniques to produce the thermally formed substrates using graphite mandrels. The graphite is relatively easy to machine into the desired shape and better matches the thermal expansion properties of the glass. Its primary disadvantage is that the graphite can only be used in an inert atmosphere oven to avoid premature aging of the material. We will also investigate a new concept for replicated optics introduced by our team member at MIT: Viscous Electrostatic Capacitor Replicated Optics for X-rays (VELCRO-X). Our goal is to replicate the surface figure of a master optic onto the microsheet glass with $\sim 1 \mu \mathrm{m}$ accuracy, in order to produce lightweight mirrors with $\leq 5$ " resolution. The fabrication process involves first placing an electric charge on the surfaces of both a glass substrate and a master. The resulting attractive force between substrate and master will ensure full conformance during the forming process, and the surface charge on the glass also prevents creases or ripples. 

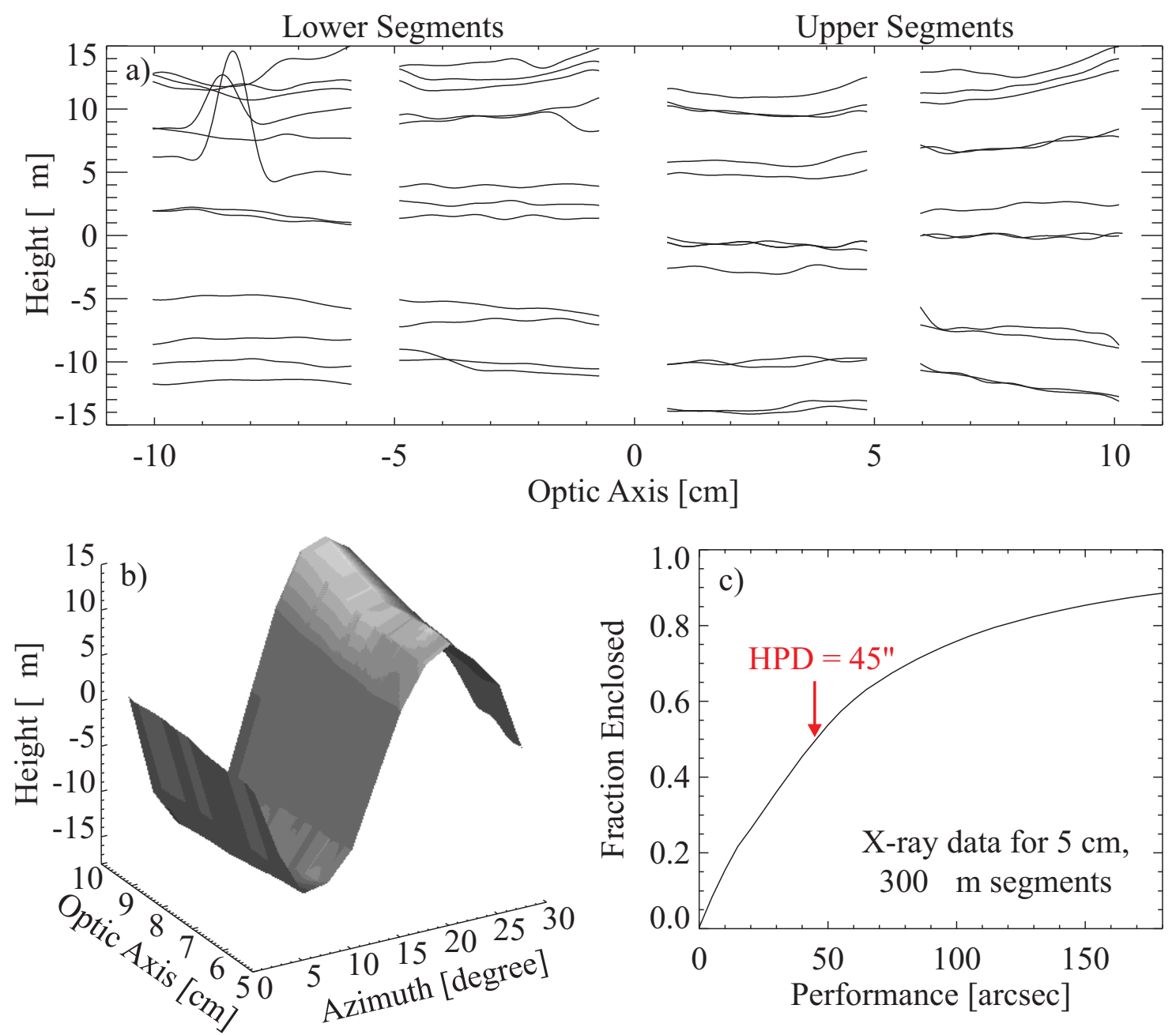

Figure 6. a) LVDT axial scans performed every $2.5^{\circ}$ for a set of two segments, $300 \mu \mathrm{m}$ thick and $5 \mathrm{~cm}$ long, which form a two-bounce section. b) The surface of uppermost segment (i.e., leftmost in the previous plot) is plotted in three-dimensions. c) From the image obtained from the X-ray data, the fraction of enclosed energy is plotted versus performance for the entire optic composed of 4 segments, and the HPD of $45 \pm 5$ " is indicated.

\section{ACKNOWLEDGMENTS}

This technical progress described in this paper could not have been made without the expert and tireless work of our technical staff. In particular, Marcela Stern was essential in defining the slumping techniques. This work is supported by a NASA grant to Columbia University: NAG5-5260 (Thermally Formed Optics for X-Ray and Gamma-Ray Astronomy), and by NASA grants to the California Institute of Technology with subawards to Columbia: Caltech No. 1019776 (High Energy Focussing Telescope) and Caltech No. 1046806 (Critical Technologies for theConstellation Hard X-Ray Telescope).

\section{REFERENCES}

1. P. Serlemitsos et al., "The x-ray telescope on board ASCA," PASJ 47(1), pp. 105-114, 1995.

2. P. Serlemitsos, "Foil X-Ray Mirrors for Astro-E," American Astronomical Society Meeting 30, pp. 1373-+, Dec. 1998. 
3. N. Westergaard, J. Polny, F. Christensen, H. Noergaard-Nielsen, and H. Schnooper, "Optical measurements of the xspect mirrors and the assembled mirror modules for the sodart x-ray telecope on the spectrum-xgamma satellite," Proc. SPIE 3113, p. 458, 1997.

4. J. Polny, N. Westergaard, F. Christensen, H. Noergaard-Nielsen, and H. Schnooper, "Production, assembly, and alignment of the xspect mirror modules for the sodart x-ray telescope on the spectrum roetgen gamma satellite," Proc. SPIE 3113, p. 349, 1997.

5. B. Aschenbach et al., "Imaging performance of the XMM-Newton x-ray telescopes," Proc. SPIE 4012, pp. 731-739, 2000 .

6. M. Weisskopf et al., "Chandra X-ray Observatory (CXO): overview," Proc. SPIE 4012, pp. 2-16, 2000.

7. D. L. Windt, J. Koglin, C. J. Hailey, E. Ziegler, F. E. Christensen, and F. A. Harrison, "Hard x-ray multilayer structures optimized for use above $100 \mathrm{kev}$," Proc. SPIE 4851(70), 2002.

8. C. Hailey, S. Abdali, F. Christensen, W. Craig, T. Decker, F. Harrison, and M. Jimenez-Garate, "Substrates and mounting techniques for the high-energy focusing telescope (heft)," Proc. SPIE 3114, p. 535, 1997.

9. W. Zhang private communication.

10. R. Petre et al. Proc. SPIE 2766, p. 11, 1999.

11. R. Petre et al., "Development of segmented x-ray mirrors for constellation-x," Proc. SPIE 4012, p. 370, 2000 .

12. J. Koglin, F. Christensen, J. Chonko, W. Craig, T. Decker, C. Hailey, F. Harrison, C. Jensen, M. Sileo, D. Windt, and H. Yu, "Development and production of hard x-ray multilayer optics for heft," Proc. SPIE 4851(67), 2002.

13. W. Craig, F. Christensen, T. Decker, C. Hailey, F. Harrison, R. Hill, M. Jimenez-Garate, P. Mao, and S. Schindler, "Hard x-ray optics for the heft balloon-borne payload: prototype design and status," Proc. SPIE 3445, p. 112, 1998.

14. W. Craig, C. Hailey, M. Jimenez-Garate, D. Windt, F. Harrison, P. Mao, F. Christensen, and A. Hussain Optics Express 7, p. 178, 2000.

15. F. Christensen, W. Craig, D. Windt, M. Jimenez-Garate, C. Hailey, F. Harrison, P. Mao, J. Chakan, E. Ziegler, and V. Honkin NIM A 415, p. 572, 2000.

16. F. Christensen, J. Chakan, F. Harrison, S. Boggs, P. Mao, T. Prince, W. Craig, C. Hailey, and D. Windt, "Grazing incidence optics designs for future gamma-ray missions," Proc. SPIE 4012, p. 278, 2000.

17. M. Jimenez-Garate, W. Craig, C. Hailey, F. Christensen, and A. Hussain Opt. Eng. 39(11), p. $2982,2000$. 